\title{
Ruptures et transversalités entre agriculture biologique et agriculture de conservation
}

Ruptures and transversality between organic agriculture and conservation agriculture

\section{Philippe Fleury, Carole Chazoule et Joséphine Peigné}

\section{(2) OpenEdition}

\section{Journals}

Édition électronique

URL : http://journals.openedition.org/economierurale/4247

DOI : $10.4000 /$ economierurale.4247

ISSN : 2105-2581

\section{Éditeur}

Société Française d'Économie Rurale (SFER)

Édition imprimée

Date de publication : 20 janvier 2014

Pagination : $95-112$

ISSN : 0013-0559

Référence électronique

Philippe Fleury, Carole Chazoule et Joséphine Peigné, « Ruptures et transversalités entre agriculture biologique et agriculture de conservation », Économie rurale [En ligne], 339-340 | janvier-mars 2014, mis en ligne le 20 janvier 2016, consulté le 19 avril 2019. URL : http://journals.openedition.org/ economierurale/4247 ; DOI : 10.4000/economierurale.4247 


\section{Ruptures et transversalités entre agriculture biologique et agriculture de conservation}

Philippe FLEURY, Carole CHAZOULE •ISARA-Lyon, Département Agriculture, Systèmes alimentaires et Territoires, Laboratoire d'Études Rurales, fleury@isara.fr, chazoule@isara.fr Joséphine PEIGNÉ•ISARA-Lyon, Département Agroécologie et Environnement, peigne@isara.fr

Cet article analyse les transversalités et les ruptures, entre deux modèles, l'agriculture biologique et l'agriculture de conservation. II s'appuie sur des entretiens semi-directifs auprès d'agriculteurs. Ces deux agricultures restent des modèles différents. Pour autant il existe des formes d'hybridation dans les pratiques de conduite des cultures. Les agriculteurs expérimentent dans leur exploitation, échangent et apprennent dans leur monde, mais ne s'y limitent pas. Agriculteurs biologiques et de conservation se rencontrent de plus en plus et ont des lectures communes. Cette hybridation technique est tout autant, sinon plus, un processus horizontal impliquant les agriculteurs et parfois des conseillers agricoles, que le résultat d'incitations institutionnelles et d'actions de diffusion.

MOTS-CLÉS : agriculture biologique, agriculture de conservation, identités professionnelles, réseau socio-technique, pratiques et référentiels des agriculteurs

\section{Ruptures and transversality between organic agriculture and conservation agriculture}

This paper analyses the transversal components and the ruptures between two models, namely organic agriculture and conservation agriculture. The results come from semi-structured interviews with farmers. Organic agriculture and conservation agriculture are remaining different models related to different values. However hybridization in the practices of crop management exists. Farmers are experimenting new techniques on their farm and sharing experiences in their own community. But they are not restricted to this, organic and conservation farmers meet increasingly and have common readings. This process of technical hybridization is equally, if not more, a process involving farmers and sometimesagricultural advisers than the result of institutional incentives and dissemination actions. (JEL: Q15, Q16, Q20).

KEYWORDS: organic agriculture, conservation agriculture, professional identities, sociotechnical networks, practices and referentials of farmers

es analyses des mouvements de diffé-renciation de modèles d'agriculture se sont majoritairement attachées à caractériser leurs spécificités en termes de valeurs, de pratiques et de réseaux : agriculture durable, agriculture raisonnée, agriculture biologique, agriculture de conservation, etc. (Goulet, 2010 ; Sylvander et al., 2006 ; Van Dam et al., 2009). Les agriculteurs engagés dans ces dynamiques revendiquent d'ailleurs leurs différences et portent souvent très fort leur sentiment d'appartenance à un groupe. Nous adoptons ici une perspective différente et l'objet de cet article est d'analyser les dimensions transversales, tout autant que les ruptures, entre deux agricultures considérées comme segmentées, agriculture biologique et agriculture de conservation.

\section{Les fondements de l'agriculture biologique et de l'agriculture de conservation}

Agriculture biologique et de conservation invoquent toutes les deux le respect $\mathrm{du}$ fonctionnement naturel du sol et plus généralement le respect de l'environnement. Mais cette référence commune se traduit par des choix techniques différents. 
L'agriculture biologique est un système agricole alternatif à l'industrialisation de l'agriculture né au début du $\mathrm{XX}^{\mathrm{e}}$ siècle. Elle est progressivement sortie de sa marginalité et est aujourd'hui définie et réglementée au niveau européen. Les derniers règlements européens qui la concernent précisent le rôle sociétal de l'agriculture biologique et les règles applicables à la production, à l'étiquetage et au contrôle des produits biologiques (Règlement (CE) $\mathrm{n}^{\circ}$ 834/2007 du 28 juin 2007 du Conseil de l'Union européenne relatif à la production biologique et à l'étiquetage des produits biologiques et règlement (CE) $n^{\circ} 889 / 2008$ du 5 septembre 2008 de la Commission des communautés européennes qui précise les modalités d'application du règlement de 2007). Un point central de la réglementation biologique est l'interdiction de l'usage d'engrais chimiques, de pesticides de synthèse et d'organismes génétiquement modifiés (OGM). Pour produire, l'agriculture biologique s'appuie sur le recyclage des matières organiques, fumiers et lisiers des animaux, la préservation de la fertilité biologique du sol, des techniques de culture peu exigeantes en intrants et la lutte biologique.

Les pratiques essentielles de l'agriculture de conservation sont la perturbation minimale du sol avec l'abandon du labour, la couverture permanente par de la végétation, des rotations culturales adaptées. Le terme de techniques culturales simplifiées (TCS) et plus récemment celui de travail sans labour (TSL) sont employés pour caractériser les techniques de travail du sol de l'agriculture de conservation. À la différence de l'agriculture biologique, l'agriculture de conservation a recours aux pesticides de synthèse, en particulier à un herbicide, le glyphosate, principe actif du Roundup de Monsanto. Goulet (2010) explique comment en une dizaine d'années praticiens et défenseurs de l'agriculture de conservation ont donné corps à un modèle d'agriculture partageant des pratiques, des valeurs et un discours, qu'il apparente à une communauté de pratiques (Wenger, 2005) : « Le non-travail du sol et la compréhension par l'agriculteur des mécanismes à l'œuvre dans cet élément deviennent les piliers d'une nouvelle agriculture. » L'agriculture de conservation peut donc se définir par un retrait, celui du labour (Goulet, 2008), comme l'agriculture biologique est l'agriculture du retrait des engrais et des phytosanitaires de synthèse.

Les relations entre agriculture de conservation et agriculture biologique ont été souvent conflictuelles. Longtemps, les défenseurs de l'agriculture de conservation se sont attachés à affirmer leur différence vis-à-vis de l'agriculture biologique (Goulet, 2010), comme les agriculteurs biologiques défendent le labour qui est pour eux un outil important pour la maîtrise des adventices. Ces deux formes d'agriculture qui revendiquent chacune de pratiquer une production respectueuse de l'environnement s'opposent donc sur les moyens utilisés : ne pas travailler le sol pour préserver cet écosystème en agriculture de conservation versus ne pas utiliser d'intrants de synthèse en agriculture biologique. Mais les temps changent. Un éditorial récent d'une revue de référence en agriculture de conservation, TCS : Techniques Culturales Simplifiées, affirme que l'agriculture biologique et de conservation sont des approches complémentaires et convergentes (Thomas, 2009). De l'autre côté, certains agriculteurs biologiques s'intéressent de plus en plus près aux TCS. Des agronomes travaillent sur les conditions et les limites de l'adaptation des techniques sans labour en agriculture biologique (Peigné et al., 2007) et plus récemment encore le Réseau mixte technologique développement de l'agriculture biologique (RMT DevAB, 2009) a publié une fiche intitulée : «Cultiver sans labour en agriculture biologique ». Même si les difficultés et l'incapacité actuelle à en généraliser l'emploi en 
agriculture biologique sont clairement décrites, le non-labour, la marque de fabrique de l'agriculture de conservation, devient une technique envisageable en agriculture biologique. Ces constats nous amènent à formuler l'hypothèse de l'émergence d'un processus d'hybridation technique entre agriculture biologique et de conservation, processus au cours duquel des techniques s'emprunteraient d'un monde à l'autre, mais où les référentiels et les valeurs, des uns et des autres bougeraient également.

\section{Une analyse des processus d'hybridation technique}

Notre article s'appuie sur un travail d'enquêtes auprès d'agriculteurs de la Région Rhône-Alpes. Pour analyser les transformations dans les ruptures et les transversalités entre ces deux agricultures, et instruire notre hypothèse, nous organisons notre analyse sous un double angle : celui des techniques et des pratiques d'une part et d'autre part celui des valeurs et modèles d'agriculture auxquels les agriculteurs se réfèrent. Nous mettons ensuite ces pratiques et discours du terrain en perspective avec les visions officielles portées par les organisations qui concourent au développement, à la défense et à la communication de l'agriculture biologique et de l'agriculture de conservation. Notre ambition est d'éclairer l'ampleur de ce processus d'hybridation technique et d'en identifier les facteurs déterminants (actions de communication, politiques publiques, protagonistes du processus) : qui sont les agriculteurs biologiques cherchant à mobiliser des techniques de l'agriculture de conservation ; et inversement, qui sont les agriculteurs de conservation s'intéressant aux techniques de l'agriculture biologique ? Quels sont leurs motivations et leurs objectifs ? Ces changements techniques sont-ils d'abord le produit d'activités de diffusion et d'information ou reposent-ils aussi sur des interactions dans lesquelles agriculteurs biologiques et de conservation prennent une part active ? Dans quels réseaux les agriculteurs et les autres parties prenantes s'inscrivent-ils? Peut-on parler d'hybridation technique, et si oui, peut-on repérer derrière celle-ci un rapprochement dans les identités professionnelles et les modèles d'agriculture ? Dans la suite de cet article, après avoir précisé notre méthode de travail, nous présentons et discutons nos résultats.

\section{Méthodes et concepts}

\section{Plusieurs sources d'information}

Notre travail d'enquête s'appuie sur des entretiens semi-directifs auprès d'agriculteurs biologiques et d'agriculteurs de conservation, mais aussi auprès de chercheurs et d'acteurs du développement de l'agriculture de la Région Rhône-Alpes. Pour les enquêtes auprès des agriculteurs nous avons utilisé un guide d'entretien comprenant six thèmes : parcours professionnel et personnel, description générale de l'exploitation, pratiques de conduite des cultures et de travail du sol, représentation du métier, visions de l'agriculture biologique et de l'agriculture de conservation et enfin réseaux professionnels. Ces entretiens ont été intégralement retranscrits avant de réaliser une analyse de contenu de chaque entretien pour chacun des six thèmes de la grille d'entretien (Bardin, 2007). Nous avons interviewé huit agriculteurs biologiques et dix agriculteurs se réclamant de l'agriculture de conservation ou tout au moins mobilisant ses techniques. De façon complémentaire nous avons rencontré deux chercheurs et deux conseillers en agriculture biologique qui tous s'intéressent à la mise au point et à la diffusion des techniques de l'agriculture de conservation. Les entretiens ont porté sur l'adaptation de ces techniques aux conditions de l'agriculture biologique, sur leurs relations professionnelles avec les 
agriculteurs et sur la vision qu'ils ont de la façon dont les agriculteurs s'informent et produisent des connaissances. Enfin, nous avons également participé à de nombreuses journées techniques, formations, visites d'exploitations et de parcelles, démonstrations de matériels et de techniques de travail simplifié du sol, à la fois dans les mondes de l'agriculture biologique et de l'agriculture de conservation. Nous avons été très attentifs aux sujets techniques discutés, à la façon dont ceux-ci sont abordés (témoignages de terrain, résultats d'essais, interventions d'experts), aux relations pédagogiques qui s'établissent entre les participants (démarche magistrale ou participative, types de savoirs convoqués et absents (scientifiques et profanes)). Cette activité d'observation participante nous a permis de préciser les réseaux et les formes de partage des connaissances privilégiés en agriculture biologique et de conservation.

Nous nous sommes enfin appuyés sur des sources écrites. Notre objectif était de faire le parallèle entre pratiques et conceptions des agriculteurs et discours officiels afin de repérer une éventuelle diversité non médiatisée. Nous nous sommes intéressés aux organisations qui concourent au développement, à la défense et à la communication sur l'agriculture biologique (Fédération nationale des agriculteurs biologiques des régions de France (FNAB), Agence Bio, International federation of organic agriculture movements (IFOAM), Institut technique de l'agriculture biologique (ITAB)) et sur l'agriculture de conservation (Agriculture de conservation.com (A2C) - portail des agricultures écologiquement intensives - et de la revue Techniques culturales simplifiées (TCS), Association pour la promotion d'une agriculture durable (APAD), Institut de l'agriculture durable (IAD), BASE : à l'origine Bretagne, agriculture sol et environnement et qui signifie aujourd' hui Biodiversité agriculture sol et environnement,
European conservation agriculture federation (ECAF), Fondation nationale pour une agriculture de conservation (FNACS)). Nous avons visité le site internet de ces organisations et analysé leurs publications principales de façon à identifier les éléments qu'elles mettent en avant pour définir leur agriculture et ses performances environnementales. Un regard particulier a été porté sur les techniques de travail du sol et sur les éventuels discours concernant l'agriculture biologique dans les organisations de l'agriculture de conservation et inversement.

\section{Analyse des données et concepts}

L'analyse des informations de l'enquête auprès des agriculteurs a eu pour finalité de classifier les agriculteurs interviewés selon trois modalités :

- la diversité des modèles d'agriculture auxquels se réfèrent agriculteurs biologiques et de conservation ;

- la façon dont ils adaptent leurs pratiques et mobilisent les techniques culturales simplifiées ;

- les réseaux professionnels dans lesquels ils s'insèrent.

C'est une approche de la diversité des conceptions de leur activité par les agriculteurs et de leurs visions de l'excellence professionnelle (Lémery, 2003 ; Lémery et al., 2005) en fonction desquelles ils s'orientent et orientent leurs choix techniques. Ceci nous a donné à voir une diversité autant au sein du groupe des agriculteurs biologiques que dans celui des agriculteurs de conservation. Nous repérons ainsi différentes formes d'agriculture qui conviennent, différentes façons d'envisager le métier qui finalement constituent un cadre dans lequel les agriculteurs envisagent l'opportunité de mobiliser telle ou telle nouvelle technique. Nous voyons ainsi comment les agriculteurs considèrent comme possibles ou non, testent et emploient différemment et plus ou moins 
systématiquement les techniques culturales simplifiées.

Le concept de référentiel (Muller, 1985) nous permet d'analyser les liens entre conceptions repérées sur le terrain et discours portés par les organisations officielles. De façon schématique, un référentiel est un cadre interprétatif par rapport auquel des individus, ou un groupe, orientent leurs actions et défendent leurs intérêts. Pour cela, ils s'appuient sur des stratégies d'argumentation destinées à rendre légitime leur référentiel, les valeurs, normes et relations causales de leur système d'explication du monde (Muller, 2005). Muller distingue deux types de référentiels : le référentiel global correspondant à la vision dominante du monde à une époque (le néo-libéralisme est un référentiel global) et les référentiels sectoriels rendant compte des représentations et principes d'action d'un secteur d'activité. Dans le secteur agricole, on peut citer par exemple le référentiel technique qui a prévalu durant la période de modernisation de l'agriculture après la Seconde Guerre mondiale. Musselin (2005) discute la notion de référentiel et montre l'intérêt de s'intéresser aussi à la diversité des représentations des acteurs et aux processus de leurs transformations. Ceci lui permet de saisir « l'action en train de se faire » et d'aller au-delà de l'analyse des discours institutionnels, des programmes et des mesures de l'action publique. Comme le propose Musselin (2005), nous mobilisons donc le concept de référentiel pour analyser les similitudes et les dissemblances entre les référentiels sectoriels des organisations de l'agriculture biologique et de conservation et la diversité des représentations des agriculteurs.

Nous avons enfin attaché une importance particulière aux réseaux professionnels, aux modes d'information et de production de connaissances mobilisés par les agriculteurs et aux formes émergentes d'échanges entre agriculteurs biologiques et de conservation. Ceci nous permet d'instruire l'hypothèse d'une interconnexion potentielle, d'apprentissages mutuels et d'échanges entre agriculteurs biologiques et agriculteurs de conservation

\section{Résultats}

Diversité des conceptions et des pratiques

La typologie des agriculteurs que nous avons élaborée permet de qualifier la façon dont les agriculteurs mobilisent, dans leur exploitation, les techniques de travail simplifiées du sol et les insèrent dans leur modèle professionnel. Nous avons identifié quatre groupes (tableau 1), mais être agriculteur biologique ou ne pas l'être reste bien une distinction centrale. Regarder vers l'agriculture de conservation et ses techniques quand on est agriculteur biologique, ce n'est pas pour autant adhérer à ce modèle d'agriculture. De façon équivalente, s'intéresser aux techniques de désherbage mécanique de l'AB ne veut pas dire «tomber dans le bio », comme l'exprime un agriculteur de conservation. Le texte suivant présente ces différentes logiques.

- Agriculteurs biologiques :

- Groupe 1 : L'autonomie du système bio polyculture-élevage comme modèle.

- Groupe 2 : La performance technique et la bio-intensive comme pratique.

- Agriculteurs de conservation :

- Groupe 3 : L'agriculture de conservation comme appartenance.

- Groupe 4 : Agriculture de conservation comme référence à des techniques. 
Ruptures et transversalités entre agriculture biologique et de conservation

Tableau 1. Modèles professionnels en agriculture biologique et techniques de travail du sol

\begin{tabular}{|c|c|c|c|c|}
\hline $\begin{array}{l}\text { Modèle de } \\
\text { référence }\end{array}$ & \begin{tabular}{|c|} 
Groupe 1 : L'autono- \\
mie du système bio \\
polyculture-élevage \\
comme modèle
\end{tabular} & $\begin{array}{l}\text { Groupe } 2 \text { : } \\
\text { La performance tech- } \\
\text { nique et la bio-inten- } \\
\text { sive comme pratique }\end{array}$ & $\begin{array}{c}\text { Groupe } 3 \text { : Agricul- } \\
\text { ture de conserva- } \\
\text { tion comme appar- } \\
\text { tenance }\end{array}$ & $\begin{array}{c}\text { Groupe } 4 \text { : Agricul- } \\
\text { ture de conservation } \\
\text { comme référence à } \\
\text { des techniques }\end{array}$ \\
\hline $\begin{array}{l}\text { Nombre } \\
\text { d'agriculteurs } \\
\text { enquêtés }\end{array}$ & 4 & 4 & 4 & 6 \\
\hline $\begin{array}{l}\text { Caracté- } \\
\text { ristiques } \\
\text { techniques des } \\
\text { exploitations }\end{array}$ & $\begin{array}{l}\text { Polyculture-élevage } \\
\text { Parfois vente di- } \\
\text { recte, exploitations } \\
\text { de taille variable }\end{array}$ & $\begin{array}{l}\text { Grandes cultures } \\
\text { dominantes, peu ou } \\
\text { pas d'élevage, pas } \\
\text { de vente directe, } \\
\text { exploitations de taille } \\
\text { variable }\end{array}$ & $\begin{array}{l}\text { Grandes exploi- } \\
\text { tations ( } 80 \text { à } 120 \\
\text { hectares), en } \\
\text { grandes cultures, } \\
\text { mais parfois en } \\
\text { polyculture-élevage }\end{array}$ & $\begin{array}{l}\text { Exploitations de taille } \\
\text { variable, en grandes } \\
\text { cultures comme en } \\
\text { polyculture-élevage }\end{array}$ \\
\hline $\begin{array}{l}\text { Techniques de } \\
\text { travail du sol }\end{array}$ & $\begin{array}{l}\text { Labour sur l'en- } \\
\text { semble des parcelles } \\
\text { cultivées }\end{array}$ & $\begin{array}{l}\text { Non-labour sur cer- } \\
\text { taines parcelles }\end{array}$ & $\begin{array}{l}\text { Abandon de tout } \\
\text { labour. Maîtrise } \\
\text { progressive des dif- } \\
\text { férentes techniques } \\
\text { de l'agriculture de } \\
\text { conservation }\end{array}$ & $\begin{array}{l}\text { Non-labour sur tout } \\
\text { ou partie de l'exploi- } \\
\text { tation }\end{array}$ \\
\hline $\begin{array}{l}\text { Transversa- } \\
\text { lités entre } \\
\text { agricultures } \\
\text { biologique et } \\
\text { de conserva- } \\
\text { tion }\end{array}$ & $\begin{array}{l}\text { Le cumul du non-la- } \\
\text { bour et de l'absence } \\
\text { d'herbicide n'est pas } \\
\text { possible aujourd'hui }\end{array}$ & $\begin{array}{l}\text { Recherche d'innova- } \\
\text { tions en s'appuyant } \\
\text { sur les techniques } \\
\text { de l'agriculture de } \\
\text { conservation }\end{array}$ & $\begin{array}{l}\text { L'agriculture } \\
\text { biologique source } \\
\text { de solutions pour } \\
\text { un nouveau défi : } \\
\text { réduire l'usage des } \\
\text { herbicides }\end{array}$ & $\begin{array}{l}\text { L'agriculture } \\
\text { biologique est une } \\
\text { autre agriculture qui } \\
\text { n'intéresse pas }\end{array}$ \\
\hline $\begin{array}{l}\text { Réseaux } \\
\text { professionnels }\end{array}$ & $\begin{array}{l}\text { Pairs aux niveaux } \\
\text { local (agriculteurs } \\
\text { biologiques ou pas) } \\
\text { et départemental } \\
\text { (agriculteurs biolo- } \\
\text { giques). } \\
\text { Techniciens du } \\
\text { développement, } \\
\text { chercheurs }\end{array}$ & $\begin{array}{l}\text { Pairs du local au } \\
\text { national (agriculteurs } \\
\text { biologiques et de } \\
\text { conservation). } \\
\text { Journées techniques. } \\
\text { Techniciens du déve- } \\
\text { loppement, chercheurs }\end{array}$ & $\begin{array}{l}\text { Pairs du local au } \\
\text { national (agricul- } \\
\text { teurs de conser- } \\
\text { vation, parfois } \\
\text { biologiques). } \\
\text { Journées tech- } \\
\text { niques. Techniciens } \\
\text { spécialisés. } \\
\text { Revues, forums } \\
\text { de discussion sur } \\
\text { l'agriculture de } \\
\text { conservation }\end{array}$ & $\begin{array}{l}\text { Pairs au niveau } \\
\text { local (agriculteurs de } \\
\text { conservation ou pas). } \\
\text { Parfois journées } \\
\text { techniques. } \\
\text { Techniciens du déve- } \\
\text { loppement }\end{array}$ \\
\hline
\end{tabular}

Source : les auteurs.

Groupe 1 : L'autonomie du système bio polyculture-élevage comme modèle

Le modèle d'agriculture idéal pour ces agriculteurs est celui du système biologique polyculture-élevage, même si parfois la réalité de l'exploitation s'en éloigne partiellement avec une proportion importante de cultures. La combinaison de productions végétales et de l'élevage est pour eux la garante de l'autonomie de l'exploitation, les animaux apportant les fumiers aux productions végétales qui assurent l'autonomie alimentaire des animaux. Le passage au bio est souvent ancien, il est la traduction d'une prise de conscience qui a poussé ces agriculteurs à changer de modèle productif : " Pour moi, c'est un autre raisonnement, c'est une autre agriculture », souligne un agriculteur.

Ces agriculteurs s'inscrivent dans des réseaux professionnels de proximité, au niveau local avec leurs collègues 
agriculteurs, au niveau départemental avec les autres agriculteurs biologiques. Dans ces réseaux s'échangent et se discutent des idées, des informations sur de nouvelles pratiques que parfois ils testent sur leurs parcelles. C'est dans ces réseaux qu'ils ont eu l'occasion d'entendre parler des pratiques du non-labour. Ils gardent visà-vis de ces techniques issues d'un autre modèle productif une réelle distance. Le non-labour, trop dépendant du glysophate, n'est dès lors jamais réellement envisagé comme une pratique utilisable et encore moins généralisable sur leur exploitation. Déjà en contradiction avec le modèle professionnel auquel ils adhérent, il présente, à leurs yeux, une difficulté difficilement contournable : la gestion de l'enherbement. En effet, en agriculture biologique, cumuler non-labour et absence de produits chimiques présenterait une trop grande prise de risque. Ces techniques ne leur semblent pas encore assez maîtrisées, au cœur de trop nombreuses controverses tant dans la sphère scientifique que dans celle du conseil. Cependant, sans parler d'abandon du labour, la réduction du travail du sol, via un labour agronomique ${ }^{1}$, est une préoccupation que l'on voit émerger. Cette pratique, moins en contradiction avec leur modèle productif, plus sûre et plus stabilisée, est d'ailleurs testée sur certaines de leurs parcelles.

\section{Groupe 2 : La performance technique et la bio-intensive comme pratique}

Contrairement au cas précédent, ces exploitations, plus grandes, sont essentiellement orientées vers les grandes cultures qui représentent une part très importante de la production, voire l'unique production. Même si le respect des principes de l'agriculture biologique reste primordial,

1. Le labour agronomique est réalisé à une profondeur réduite de $15-20 \mathrm{~cm}$ et sans rasette. Ceci limite le mélange de la matière organique dans l'ensemble de la couche de sol travaillée. ces agriculteurs s'éloignent plus que les précédents de son modèle de référence (exploitation autonome, polyculture-élevage, circuits courts et liens avec le consommateur...) qui n'est d'ailleurs pas revendiqué. Ils se qualifient eux-mêmes de « bio-intensifs », du fait de l'importance des grandes cultures et de la spécialisation de leur exploitation. Dès lors, même s'ils justifient leur passage en agriculture biologique par une volonté « de ne pas empoisonner les sols et la production avec des produits chimiques », ils revendiquent tout autant de raisonner le choix de leur rotation selon des critères économiques : " On sait que dans l'idéal, il faudrait faire des rotations très longues... On sait aussi qu'en bio, il $\mathrm{y}$ a un système qui fonctionne très bien, des rotations sur 3 ans (maïs, soja, blé) et qui économiquement est beaucoup plus intéressant pour l'agriculteur que les systèmes avec des rotations de $7 / 8$ ans et des jachères de 2/3 ans. [...] Si les rotations courtes sont moins bien sur le plan agronomique, elles sont mieux sur le plan économique. » Dans ce groupe, la technicité est perçue comme un réel facteur d'excellence et de reconnaissance professionnelle. Cette recherche d'amélioration constante se traduit par la mise en place ou la participation aux réseaux, parfois d'échelle nationale, de l'agriculture biologique, mais aussi, bien qu'à un moindre degré, à ceux de l'agriculture de conservation. Ils privilégient les échanges concernant les techniques, le matériel et l'expérimentation (portée par des institutions ou par des agriculteurs).

Certaines techniques de l'agriculture de conservation sont entrées dans leur modèle productif. La réduction du travail du sol en fait partie, tout comme le semis sous couvert. Pour eux, réduire, voire à terme envisager de stopper le labour relève de la même logique de préservation des sols que celle qui consiste à réduire les intrants chimiques. La volonté de développer ces techniques les conduit cependant à exprimer des difficultés. Ils évoquent en 
particulier la prise de risque tant sur le plan technique qu'économique liée à la gestion des adventices. Le problème d'enherbement constitue ainsi la crainte majeure des exploitants, suivi directement de la question de gestion de la fertilisation : « Je connais quelqu'un qui est passé en technique sans labour, il n'a pas géré suffisamment ses engrais verts, il est arrivé au moment où il fallait semer, il avait des engrais verts qui s'étaient trop développés, ce qui fait qu'il a eu des nématodes dans ses cultures et ça a été mangé. » Ces agriculteurs insistent sur le fait que chaque étape doit être raisonnée en fonction d'un grand nombre de paramètres (sol, semences, météo, outils disponibles, etc.). Plus rien ne peut être systématique, tout doit être réfléchi : «Moi, je n'ai pas d'idéologie, je m'adapte... Dans ma rotation, j'ai 2 à 3 ans sans labour, enfin, pas qui se succèdent, mais intercalés. Donc je m'adapte à mes conditions de sol, si je juge que ce n'est pas nécessaire, que je n'ai pas trop d'enherbement. »

Pour tous, le développement des techniques culturales simplifiées en agriculture biologique se heurte à un manque de connaissances. D'une part, les recherches ne sont pas suffisamment développées et d'autre part la sphère du conseil n'est pas assez présente. Les techniques culturales simplifiées sont aussi un challenge, une occasion de mettre en place de nouvelles pratiques, de créer de nouvelles connaissances et de créer de nouveaux réseaux. Tous soulignent l'intérêt de mettre en commun leurs expériences et leurs connaissances, de créer des groupes de partage et d'échanges : «On est sur terre, on n'a pas à garder ce que l'on sait, il faut le diffuser, voilà. » Leurs emprunts techniques et leurs échanges avec les agriculteurs de conservation ne les font pas pour autant adhérer à cette agriculture. Ils sont très attachés à leur identité d'agriculteur biologique, leur conversion étant liée à une volonté de « ne pas empoisonner les sols et la production avec les produits chimiques ». Ils n'acceptent d'ailleurs pas l'appropriation actuelle du terme « conservation », car comme le dit l'un d'entre eux : «Aucun modèle d'agriculture n'est plus conservateur qu'un autre. »

\section{Groupe 3 : L'agriculture}

de conservation comme appartenance

Les agriculteurs de ce groupe se sont intéressés puis ont développé le travail sans labour pour répondre à des objectifs de gestion optimisée de leur temps de travail et de leurs ressources (sol, mais aussi investissements). Ils sont en quête d'une amélioration de l'autonomie de leur exploitation pour réduire les coûts. Leur modèle professionnel est celui de l'agriculture de conservation, mais ils insistent sur la nécessité que celuici évolue, progresse notamment vis-à-vis de l'usage du glyphosate : "Il faut lutter contre [le glyphosate et les intrants], c'est juste pour engraisser les multinationales qui n'ont que faire des agriculteurs et qui ne cherchent qu'à faire de l'argent. » Ils sont abonnés et lisent régulièrement les revues des réseaux de l'agriculture de conservation (TCS) ou d'autres qui parlent souvent de ses techniques (Cultivar). Ce sont pour eux des références techniques incontournables. Ils peuvent également être inscrits dans des réseaux sociaux sur Internet où ces questions sont discutées (site Agricool). Ils entrent alors en relation avec des agriculteurs de différentes régions. Ces forums sont des lieux où ils trouvent des réponses à certaines de leurs questions et échangent sur leurs problèmes. Enfin, ils ont suivi des formations sur les techniques de cultures simplifiées et le sans labour afin de mieux comprendre le sol et sa vie biologique. Ces agriculteurs recherchent des informations dans différents lieux et n'appartiennent pas à un seul réseau d'échanges, au contraire ils les multiplient dans les différentes sphères de l'agriculture de conservation, du conventionnel et parfois de l'agriculture biologique. 
RECHERCHES

Ce qui caractérise ces agriculteurs est un intérêt récent pour l'agriculture biologique. Tous on déjà effectué des visites dans des exploitations biologiques afin de mieux en comprendre le système technique. Certains font même partie d'un groupement où des agriculteurs conventionnels se réunissent autour de techniques innovantes et rencontrent régulièrement des agriculteurs biologiques afin de pouvoir s'inspirer de leurs techniques et ainsi limiter les intrants chimiques. Pour autant, ils n'envisagent pas de se convertir à l'agriculture biologique. Plusieurs arguments sont avancés pour justifier leur volonté de ne pas «franchir le pas ». Il s'agit soit d'un manque de connaissance du système, soit d'une crainte de devoir reprendre le labour pour maîtriser l'enherbement, soit encore du refus de la certification qu'ils jugent trop contraignante. Cependant, la justification la plus souvent évoquée est celle de la moindre connaissance du sol et de sa vie biologique par les agriculteurs biologiques : "Quand tu leur parles de la vie microbienne dans le sol, ils ne savent pas... Ils ne savent même pas pourquoi il y a des vers de terre dans la terre. » Cet objet qui leur semble moins central dans l'agriculture biologique est au cœur du raisonnement de leurs pratiques : «Moi, j'ai toujours pensé que la terre n'était pas qu'un support à céréales, à production, moi, je voulais nourrir ma terre pour travailler mon sol et le faire travailler autrement. Je voulais éviter de l'abîmer et de détruire ma vie microbienne. » Ils considèrent ces différences de connaissance et de conception du sol comme un élément discriminant entre leurs pratiques et celles des agriculteurs biologiques. Ils réfutent également aux agriculteurs biologiques le privilège de l'excellence en matière d'environnement et de pollution : " On peut faire des choses plus propres sans tomber dans le bio », et préfèrent s'inscrire dans le cadre de l'agriculture intégrée : "Je vais faire le tour des céréales pour voir s'il y a besoin de faire des rattrapages, je ne fais rien de systématique, mais si besoin je fais des rattrapages sur les mauvaises herbes des céréales. C'est pour ça moi je parle beaucoup d'agriculture intégrée. »

Tous les agriculteurs de ce groupe soulignent leur intérêt pour l'agronomie et la compréhension des mécanismes de la vie biologique du sol. C'est un élément majeur de leur identité professionnelle, acquérir des connaissances sur ces objets est pour eux un moyen de développer une forme d'excellence professionnelle, d'être parmi les meilleurs dans leur domaine. C'est la clef pour rester compétitif et assurer la pérennité de leur exploitation. Ils regrettent cependant le manque de solutions et de connaissances issues de la recherche ou des réseaux de conseil qui leur seraient utiles pour aller vers une hybridation plus forte des techniques de l'agriculture biologique et de l'agriculture de conservation.

\section{Groupe 4 : Agriculture de conservation comme référence à des techniques}

Ce groupe se différencie du précédent par son désintérêt pour l'agriculture biologique et son inscription limitée dans des réseaux d'agriculteurs. On y rencontre à la fois de grandes exploitations céréalières et des exploitations, plus petites, de polyculture-élevage. Ils pratiquent le nonlabour sur une partie de l'exploitation ou à certaines périodes (automne). Certains ont même totalement abandonné le labour. Ils évoquent différentes motivations. Les principales sont le gain de temps et la réduction des coûts de production. Tous insistent sur la dimension économique : «Au niveau économique, c'est mieux, car on passe moins de temps dans les terres, ça réduit nos charges et puis le tracteur, ça équivaut à manger du gasoil. » Comme pour le groupe précédent, la motivation agronomique est bien présente : «Nos sols sont plus vivants et il y a des vers de terre, ils résistent mieux à l'érosion. » 
Ces agriculteurs se considèrent parfois comme isolés et comme manquant de références pour développer les techniques culturales simplifiées et le semis direct : «Disons que je ne demande pas grand-chose, parce que je ne sais pas trop où trouver. Personne ne me suit dans cette voie, à la coop, il n'y a personne. » Ils disent également ne pas rechercher de contacts avec d'autres agriculteurs ou avec des experts. À la différence du groupe précédent, ils ne font ainsi pas référence aux spécialistes et aux pionniers de l'agriculture de conservation en France. Ils nous disent s'être tout simplement lancés dans le semis direct après avoir vu certains de leurs voisins ou de leurs amis le faire. Ils soulignent même parfois ne pas en avoir discuté avec eux. Ils ont progressé par tâtonnement : «Une année, j’ai essayé, mais en règle générale je ne labourais déjà presque plus... donc l'année 2002, j' ai dû encore labourer, mais que le maïs et qu'en partie, pas tout. J'ai commencé en tâtonnant. L'année d'après, j'ai fait pareil, je labourais, je ne labourais pas, je démarrais doucement, je n'ai pas tout basculé d'un coup. » S'ils ont tous participé à des journées techniques, ils l'ont fait de manière occasionnelle lorsqu'ils étaient confrontés à des problèmes. Ces journées ne se traduisent ni par la création de liens avec d'autres agriculteurs ni par l'inscription dans des réseaux où se discutent les techniques culturales simplifiées. Ils mettent en avant le manque de références techniques et l'absence de relation avec la recherche et s'interrogent sur l'intérêt de telles journées.

Bien que ces agriculteurs aient une connaissance précise du terme agriculture de conservation, ils ne s'y reconnaissent pas. Pour eux, le terme est encore trop vague et regroupe des pratiques trop différentes qu'ils n'ont pas toutes adoptées : techniques culturales simplifiées, non-labour, semis direct, semis direct sous couvert, etc. Certains ne font que du non-labour, d'autres pratiquent le non-labour pour les semis d'automne, mais pas pour ceux de printemps, d'autres encore pratiquent les semis directs sous couvert. Ici, le glyphosate pose moins question que dans le groupe précédent et son emploi se fait sans état d'âme : «On a beaucoup d'herbe, il faut la maîtriser, au début on passe beaucoup plus de Roundup que d'habitude, il faut avoir des parcelles propres pour ne pas laisser grainer. » Un autre élément fait qu'ils ne se reconnaissent pas dans l'agriculture de conservation. En effet, pour eux, se dire agriculteurs de conservation reviendrait à s'inscrire dans un groupe professionnel qu'ils trouvent trop militant « un peu comme les bios ». Ils souhaitent garder leur indépendance et pratiquer l'agriculture qu'ils « veulent », adaptée à leur exploitation, sans avoir à adhérer à un système de valeurs.

\section{Proximités et transversalités entre modèles d'agriculture}

De nombreux travaux se sont attachés à caractériser la diversité des agriculteurs ou de leurs exploitations. Il existe ainsi beaucoup de références affirmant et caractérisant la pluralité des façons de pratiquer l'agriculture biologique (Sylvander et al., 2006 ; Desclaux et al., 2009 ; Van Dam et al., 2009). La typologie que nous proposons a été construite avec une finalité différente. Il ne s'agit pas de rendre compte dans la finesse de la diversité des modèles professionnels en agriculture biologique ou en agriculture de conservation, mais d' analyser à la fois les ruptures et les proximités dans ces modèles tant dans l'agriculture biologique et dans l'agriculture de conservation qu'entre agriculture biologique et agriculture de conservation. La figure 1 situe les différentes formes d'hybridation technique entre agriculture biologique et agriculture de conservation selon l'importance et la nature des réseaux professionnels des agriculteurs. En effet, comme nous allons le voir, parmi les nombreux facteurs en jeu dans ce processus d'hybridation les réseaux des agriculteurs jouent un rôle central. 
RECHERCHES

Figure 1. Formes d'hybridation technique entre agriculture biologique et de conservation selon les réseaux professionnels des agriculteurs

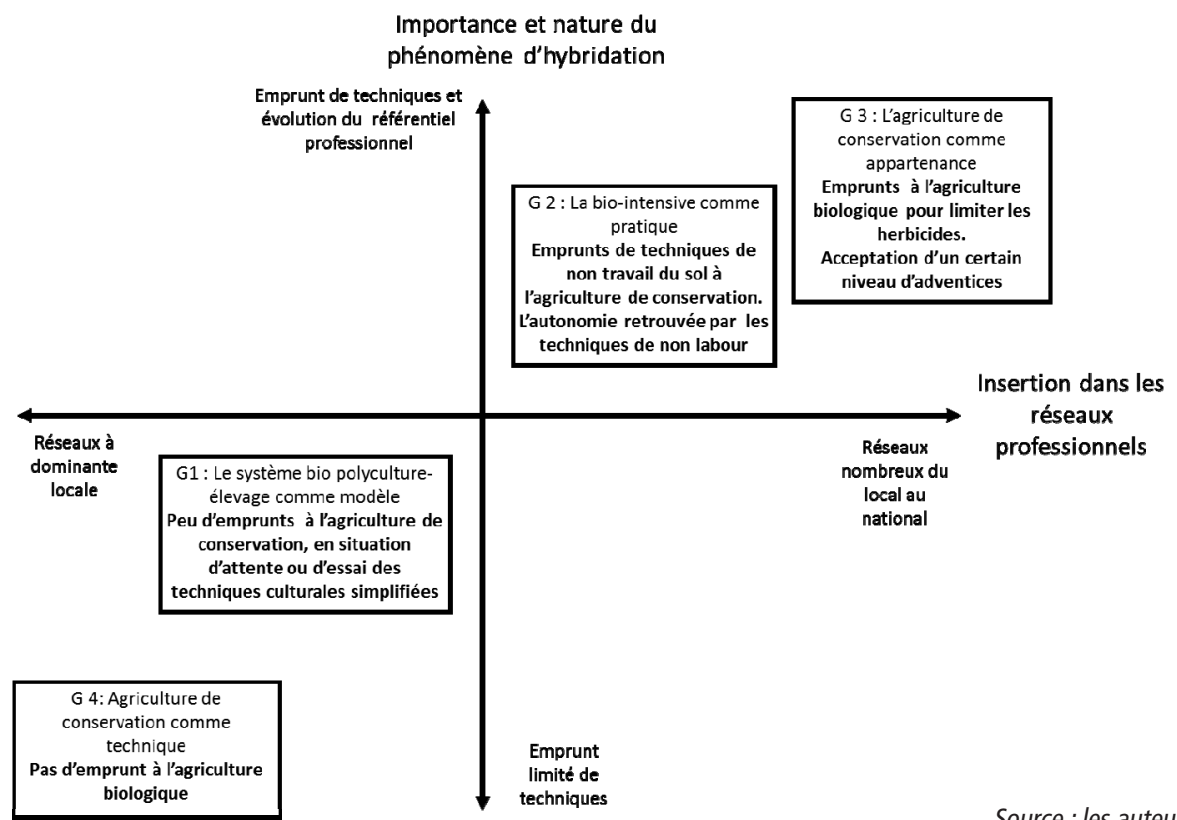

Source : les auteurs.

On le voit, le processus d'hybridation technique entre agriculture biologique et agriculture de conservation est à l'œuvre selon une intensité et des modalités diverses suivant les groupes d'agriculteurs.

Tout d'abord, les agriculteurs du quatrième groupe, « l'agriculture de conservation comme technique », ne s'intéressent pas à l'agriculture biologique et se reconnaissent également peu dans les valeurs de l'agriculture de conservation. Ce qu'ils cherchent dans celle-ci, de façon très pragmatique, ce sont certaines de ses techniques. Ces agriculteurs ne s'inscrivent pas dans les réseaux sociotechniques ayant permis de développer les techniques sans labour, ils donnent la priorité à l'apprentissage individuel et secondairement aux échanges avec des pairs proches d'eux.

Le premier groupe, constitué d'agriculteurs biologiques se caractérise par une référence forte à l'autonomie du système polyculture-élevage. Les réseaux professionnels sont locaux, ils lient des agriculteurs d'un même territoire ou d'un département. Les questions discutées dans ces réseaux sont générales et ne concernent pas spécifiquement l'enjeu technique du travail du sol. Pour ces agriculteurs biologiques, la question du travail simplifié du sol est ouverte, mais, au-delà de quelques essais ponctuels il n'y a pas encore de réelle mise en application.

Dans les groupes deux et trois, « agriculture de conservation comme appartenance » et «performance technique et biointensive comme pratique », on retrouve la volonté de comprendre comment fonctionnent les techniques de l'autre modèle. Les agriculteurs biologiques portent un intérêt aux techniques issues du modèle de l'agriculture de conservation et inversement. C'est au sein de ces deux groupes que l'on rencontre la plus forte hybridation technique et que les pratiques sont le plus en mouvement. Elles bougent et se 
complexifient en permanence. Les réseaux professionnels sont de moins en moins territoriaux, se déploient au niveau national et même international avec l'aide d'internet. On y observe tout d'abord des emprunts de techniques à l'autre modèle d'agriculture : mobilisation des techniques de travail simplifié du sol, voire abandon du labour chez les agriculteurs biologiques et inversement recours à certaines techniques de l'agriculture biologique chez les agriculteurs de conservation comme l'allongement des rotations pour limiter les adventices, l'implantation de légumineuses ou le compostage de matières organiques pour améliorer la fertilité des sols. Au-delà, on note même une évolution des référentiels, des critères d'excellence professionnels, comme l'acceptation d'une présence plus importante d'adventices pour certains agriculteurs de conservation : "Comme chez certains bios on a pas mal d'adventices, mais il faut faire avec, les accepter » ou pour les agriculteurs biologiques une vision de l'autonomie qui ne reposerait plus sur le système polyculture-élevage, mais sur une autonomie acquise à l'échelle de la parcelle en raisonnant pour cela la conduite et le choix des cultures.

\section{Discussion}

\section{Transformation des référentiels}

\section{d'action technique}

Même si certaines techniques, comme l'attention portée aux rotations, se retrouvent en agriculture biologique et de conservation, il y a une différence de fond en matière de pilier, de clef de voûte, sur lequel repose la conduite des cultures. En agriculture biologique, en réponse à l'abandon des intrants, engrais et phytosanitaires de synthèse, le labour est une condition essentielle en matière de maîtrise des adventices. En agriculture de conservation, ce sont les désherbants de synthèse, le glyphosate qui assurent cette fonction de maîtrise des adventices. Glyphosate d'une part, labour d'autre part sont au cœur des référentiels
(Muller, 1985) de l'agriculture de conservation et de l'agriculture biologique.

Les référentiels de l'agriculture de conservation et de l'agriculture biologique, comme ceux d'autres agricultures (Stassart et Jamar, 2009), se déploient en rendant visibles certains éléments comme la suppression du labour, sa couverture permanente par la végétation en agriculture de conservation ou l'utilisation de substances naturelles en agriculture biologique et en laissent d'autres dans l'ombre. En agriculture de conservation, l'élément que l'on expose le moins possible, comme l'a montré Goulet (2008) est l'usage du glyphosate et l'appui des firmes agro-chimiques aux organisations professionnelles de l'agriculture de conservation. Cela a été pour elles une condition essentielle dans la revendication de l'excellence environnementale. Pour l'agriculture biologique, il existe également un élément de son référentiel qui est peu médiatisé. Il s'agit de la répétition des opérations de travail du sol, labour, désherbage mécanique, binage. Ces opérations, alternatives à l'usage des phytosanitaires, exigeantes en temps pour l'agriculteur, en énergie et en matériel ont un coût économique et environnemental (Peigné et al., 2009).

Ces éléments peu visibles, mais pourtant essentiels, de l'agriculture biologique et de l'agriculture de conservation, apparaissent de plus en plus au grand jour. Les questions du glyphosate et des liens de l'agriculture de conservation avec l'agrochimie posées de façon marginale, par exemple dans les publications scientifiques de Goulet (2008 et 2010) se retrouvent de plus en plus audevant de la scène. Les porte-parole de l'agriculture de conservation en sont bien conscients et s'y préparent : «Afin de poursuivre le développement de l'agriculture de conservation, sans prise de risque inconsidérée, la stratégie consiste à trouver de nouveaux leviers d'actions, quitte à restreindre les usages du produit sans pour 
autant l'interdire. Le glyphosate était le pilier du semis direct, il est devenu le filet de sécurité de l'agriculture de conservation » (Archambeaud, 2011). Dans l'agriculture biologique, le débat autour du labour s'ouvre. Les promoteurs de la suppression du labour évoquent des intérêts agronomiques, écologiques (augmentation du potentiel de stockage de carbone même s'il y a aussi augmentation des émissions de protoxyde d'azote) et économiques (économies de carburant et réduction du temps de travail) (RMT DevAB, 2009).

Cette transformation des référentiels sectoriels de l'agriculture biologique et de l'agriculture de conservation n'est pas seulement portée par leurs organisations professionnelles respectives. Elle est aussi en marche chez les agriculteurs. Comme l'a montré la typologie que nous avons présentée, certains agriculteurs biologiques et de conservation contribuent, par leurs discours, les échanges qu'ils développent, au changement technique et au renouvellement des référentiels.

Pour les «bios intensifs » qui s'intéressent de près au semis direct, le modèle de l'agriculture biologique fondé sur le respect des cycles biologiques, l'autonomie vis-à-vis des firmes de l'agrochimie est une référence avec laquelle ils sont souvent confrontés. Le décalage entre le modèle productif de référence de l'agriculture biologique, le système polycultureélevage et leurs pratiques est régulièrement pointé dans les organisations biologiques : l'absence des animaux et de leur fumier nécessite plus d'intrants en particulier en matière de fertilisants azotés organiques. Pour compenser cette perte d'autonomie, les solutions proposées, comme le recours plus important aux légumineuses, répondent incomplètement aux besoins ou sont complexes à mettre en place, comme l'échange de paille contre du fumier entre exploitations de grandes cultures et exploitations d'élevage. Dans ce contexte, les techniques culturales simplifiées, leurs effets positifs sur la vie biologique du sol et sur l'autonomie azotée, sont pour ces agriculteurs des techniques intéressantes qu'ils cherchent à adapter dans leur exploitation. Ils envisagent ainsi de retrouver une nouvelle cohérence entre leurs pratiques et leurs valeurs professionnelles, celles de l'autonomie et du respect des cycles biologiques. Investir les techniques de l'agriculture de conservation, les rendre opérationnelles en agriculture biologique, c'est pour les bios intensifs, progresser dans la cohérence avec les fondements de l'agriculture biologique. Principes auxquels beaucoup sont attachés tant pour des raisons agronomiques qu'éthiques et qu'ils considèrent avoir de plus en plus de mal à maintenir dans leur système de production spécialisé.

L'intérêt des agriculteurs de conservation pour les techniques de l'agriculture biologique correspond à un cheminement différent. Il est à remettre en perspective à la fois avec la montée en puissance de la question environnementale et avec la notion d'élite technique en agriculture de conservation. C'est bien au départ pour réduire leur temps de travail et gagner en productivité que les agriculteurs se sont lancés dans les techniques sans labour, mais très vite ils se sont pris à un jeu passionnant. Celui de l'apprentissage et de la maîtrise de techniques complexes aux effets non garantis. En agriculture de conservation, on progresse pas à pas, on apprend tous les jours. Réussir ses productions en maîtrisant une combinaison de plus en plus complexe et fine de techniques est un critère d'excellence professionnelle majeur. Réduire l'usage des herbicides, c'est relever un défi supplémentaire et progresser dans cette maîtrise. Défi d'autant plus motivant à saisir que dans un contexte sociétal, mettant en avant le respect de l'environnement, l'agriculture de conservation revendique l'excellence environnementale. Pour cela, elle argumente surtout 
sur une contribution positive au changement climatique du fait d'un stockage de carbone meilleur dans les sols non labourés (Holland, 2004). Ce discours trouve ses limites et il est de plus en plus difficile pour l'agriculture de conservation de ne pas se préoccuper des produits phytosanitaires qu' elle utilise. Sur le terrain, les agriculteurs expriment très souvent le fait que leurs pratiques phytosanitaires, l'usage du pulvérisateur équipé d'un masque et d'une tenue de protection, sont fortement stigmatisés par leurs voisins. Pour les agriculteurs de conservation, regarder du côté de l'agriculture biologique pour en reprendre certains éléments techniques (compostage, successions culturales) et réduire le recours aux phytosanitaires est à la fois un défi technique motivant, mais aussi une façon de répondre à une pression environnementale tout autant locale que globale.

\section{Les facteurs de l'hybridation}

L'hybridation émergente des techniques entre agriculture biologique et agriculture de conservation est tout à la fois une affaire d'institutions et un processus horizontal dans lequel les agriculteurs prennent une part active.

Du point de vue des institutions, elle est portée par certaines organisations de l'agriculture de conservation depuis la fin des années 2000. Ces organisations, s'ouvrant aux techniques de l'agriculture biologique, sont essentiellement, pour ne pas dire seulement, animées par des agriculteurs ou des techniciens. Ainsi, Frédéric Thomas, rédacteur en chef de la revue TCS et agriculteur en Sologne, très actif et reconnu dans la mise au point et la diffusion des techniques sans labour communique sur la complémentarité entre agriculture de conservation et agriculture biologique (Thomas, 2009). En 2010, la revue TCS publie un article consacré à Joseph Pousset, agriculteur biologique pratiquant le sans labour. L'article insiste tout particulièrement sur son mode innovant de gestion des adventices en limitant, par les successions culturales, le stock semencier (Waligora, 2010). Le site, agriculture-de-conservation.com, de la revue TCS met en avant le pionnier des techniques de travail sans labour en agriculture biologique : on y trouve un article repris de la revue Alter Agri (revue technique de l'ITAB dédiée aux techniques alternatives) consacré à Friedrich et Manfred Wenz en Allemagne qui conduisent une ferme céréalière en agriculture biologique sans pratiquer de labour depuis plus de 25 ans. L'article décrit avec de nombreux détails l'histoire et les pratiques de cette exploitation. On y insiste beaucoup sur le salissement progressif des parcelles et la perte d'efficacité des herbicides, une des raisons qui ont déclenché le passage au non-labour (Leclerc et al., 2007). L'association BASE organise depuis 2011 des journées spécifiquement dédiées aux « relations entre agriculture de conservation et agriculture biologique ». Pour BASE, association dont les statuts précisent qu'elle est « indépendante de toute entreprise privée, organisation professionnelle, syndicat ou autre association » (www.asso.base.fr), ce qui est en jeu c'est de développer les réseaux de l'agriculture de conservation, de les ouvrir à l'ensemble de la profession « agriculteurs dits conventionnels ou bio, techniciens et scientifiques... » (www.agriculture-deconservation.com). D'autres organisations de l'agriculture de conservation n'ont pas de discours sur l'agriculture biologique. Il en est ainsi de l'IAD, dont les membres sont des agriculteurs, des associations de l'agriculture de conservation des sols et des entreprises dont Monsanto Agriculture France (semences et produits phytosanitaires dont le Roundup) et Syngenta Agro (semences et produits phytosanitaires). L'IAD ne parle pas de l'agriculture biologique et encore moins de sa proximité éventuelle avec l'agriculture de conservation, elle préfère poser sa démarche « rationnelle et objective » comme dépassant « les oppositions et les débats idéologiques, 
comme ceux opposant partisans de la croissance et ceux de la décroissance, libéraux et altermondialistes, industriels et bio » (www.institut-agriculture-durable.fr).

Les organisations de l'agriculture biologique ont un rôle encore plus discret dans la promotion de cette hybridation technique. Tout d'abord les organisations techniques et de la recherche-développement de l'agriculture se situent dans la sphère des techniques. Il s'agit pour elles de mettre au point des techniques de travail simplifié du sol adaptées à l'interdiction en agriculture biologique des pesticides de synthèse. Il en va ainsi des travaux de l'ISARA-Lyon, de l'ITAB et du RMT DevAB. Les organisations de défense et de représentation politique de l'agriculture biologique ont quant à elles un discours prudent et il ne s'agit en aucun cas pour elles d'évoquer une quelconque parenté entre agriculture biologique et agriculture de conservation. Ainsi, l'IFOAM dans un texte de positionnement oppose la focalisation de l'agriculture de conservation sur une seule technique à l'agriculture biologique qui se fonde sur une approche globale mobilisant un ensemble de techniques et qui s'appuie sur un système de certification et de contrôle facilitant son pilotage et son évaluation environnementale (IFOAM, 2004). Elle insiste sur le rôle de l'agriculture biologique qui est d'intégrer le travail du sol sans labour en évitant ses effets négatifs (Kotschi et Müller-Sämann, 2004).

Enfin, la FAO promeut à la fois, mais par des voies différentes, l'agriculture de conservation et l'agriculture biologique. Elle se limite à une information sur la différence entre agriculture de conservation et agriculture biologique et n'évoque pas de complémentarité entre elles. Ainsi sur le site internet consacré à l'agriculture de conservation elle s'en tient à un positionnement des mérites de l'agriculture de conservation par rapport à l'agriculture conventionnelle : «En règle générale, les cultivateurs qui pratiquent une agriculture de conservation utilisent moins d'intrants chimiques que les agriculteurs traditionnels et la quantité de produits chimiques employés tend à diminuer au fil des années » (FAO, 2006).

Comme nous l'avons détaillé dans la partie résultats de cet article, l'hybridation des techniques entre agriculture biologique et agriculture de conservation est donc bien d'abord un processus horizontal de terrain (Ansaloni et Fouilleux, 2006). Ce processus de terrain est multiforme, on $\mathrm{y}$ voit des agriculteurs biologiques et des agriculteurs de conservation se rencontrer dans des journées techniques, échanger entre voisins, discuter dans des forums internet, expérimenter ou importer dans leur exploitation des techniques nouvelles. Les modalités de cette hybridation sont nombreuses comme les raisons et les motivations des agriculteurs qui s'y engagent : mobilisation de techniques plus adaptées à l'organisation technico-économique de l'exploitation, recherche de l'excellence professionnelle ou d'une cohérence entre pratiques et vision du métier, volonté d'anticiper des injonctions environnementales (Lamine, 2011) comme inévitables dans les politiques publiques à venir, etc. Ce processus horizontal de terrain est d'ailleurs un peu plus ancien que la médiatisation des complémentarités entre agriculture de conservation et biologique dans quelques organisations de l'agriculture de conservation. Les agriculteurs enquêtés nous parlent de premiers échanges, de réflexions, de visites de forums internet dès 2005 ou plus fréquemment à partir de 2007 ou 2008, alors que l'ouverture officielle de tribunes de l'agriculture de conservation aux techniques biologiques ne s'est faite qu'à partir de 2009.

C'est bien un double processus que nous observons, les impacts des activités de communication de certaines organisations de l'agriculture de conservation et 
des organisations techniques de l'agriculture biologique se cumulent avec le rôle actif d'agriculteurs à la recherche de nouvelles cohérences techniques. Les deux branches de ce processus se nouent et se reconnaissent mutuellement. D'un côté, les organisations légitiment et médiatisent les échanges entre agriculteurs en mettant en exergue leurs savoirs et savoir-faire adaptés à la diversité des conditions locales. De l'autre, les agriculteurs de conservation, mais aussi les agriculteurs biologiques qui tous se sont longtemps considérés comme les oubliés de la recherche et du développement agricole (Goulet, 2008 pour les agriculteurs de conservation, Van Dam et al., 2009 pour les agriculteurs biologiques), appellent à intensifier les recherches ou le conseil technique et lorsqu'ils en ont l'occasion prêtent volontiers une parcelle pour qu'un organisme de recherche ou de développement puisse y conduire des expérimentations.

\section{Perméabilités, complémentarités et compétition}

La démarcation entre agriculture biologique et agriculture de conservation reste. S'intéresser à des techniques, en tester et en emprunter certaines dans son exploitation, ce n'est pas adhérer à un autre modèle professionnel.

Même si les échanges entre ces agricultures se développent, l'attachement identitaire et technique des agriculteurs à un modèle reste fort. L'agriculture biologique est un modèle dont la légitimité s'inscrit dans le champ sociétal ; elle est une référence pour de nombreux consommateurs et citoyens comme pour des environnementalistes et des collectivités territoriales qui cherchent à développer l'agriculture biologique pour résoudre un problème de qualité de l'eau, pour fournir leur restauration collective en produits biologiques, etc. (Fleury, 2011). C'est une différence forte avec l'agriculture de conservation qui jusqu'alors reste envisagée comme un ensemble de techniques et comme un modèle d'agriculture dont la légitimité se construit dans le champ sectoriel et professionnel. Cependant, plusieurs agriculteurs de conservation que nous avons rencontrés, s'appuient sur l'exemple de l'agriculture biologique, pour évoquer la perspective de démarquer leurs produits, de communiquer sur leurs pratiques auprès des consommateurs et de chercher à obtenir une plus-value qui n'existe pas aujourd'hui. Une idée, dont l'avenir nous dira si elle se concrétisera ou non.

Mais il est un sujet, celui de l'environnement, où d'ores et déjà l'agriculture de conservation s'implante, construit une légitimité, fait valoir son référentiel en se déployant tant au niveau des agriculteurs qu'au niveau institutionnel, par ses organisations ou avec le relais d'autres plus génériques (comme la FAO ou l'Association internationale pour une agriculture écologiquement intensive (AEI)). En 2010, la FAO décrit l'agriculture de conservation comme visant « des systèmes agricoles durables et rentables » et comme présentant « un grand potentiel pour tous les types d'exploitations agricoles et environnements agro-écologiques ».

L'agriculture de conservation se revendique donc comme environnementale et productive. Elle marche sur les brisées de l'agriculture biologique, en mettant en avant deux arguments où celle-ci est controversée : son niveau de production et son impact sur le changement global (Fleury, 2011). Les arguments des uns et des autres se mobilisent dans des champs qui se rapprochent : niveau de production et croissance démographique, environnement, alimentation et santé. C'est bien sur ces deux terrains, celui de la production et plus largement de la contribution à l'alimentation et celui de l'environnement qu'à l'avenir les choses semblent devoir se discuter entre agriculture biologique et de conservation. Ici, il n'est pas si facile 
RECHERCHES

de parler de complémentarité. Le débat paraît être bien plus dans l'évaluation, la comparaison et les enjeux sont ceux des soutiens de l'action publique et de l'adhésion des citoyens et des consommateurs. Ce débat est aujourd'hui ouvert aux ÉtatsUnis où plusieurs articles de presse et sites internet à destination du grand public évoquent «The Battle for the Green Label - Organic Farming Versus Conservation Agriculture » (Saikat, 2008) et interrogent des références scientifiques, pour savoir si l'agriculture de conservation est meilleure pour l'environnement que l'agriculture biologique (Layton, 2009). Ainsi, en parallèle des processus émergeant d'hybridation technique que l'on observe chez les agriculteurs, cela paraît bien être aussi une compétition politique qui s'ouvre entre ces deux modèles d'agriculture dont la reconnaissance sociale et l'accès aux soutiens publics ne peuvent plus être considérés comme acquis, mais bien à construire et à entretenir en permanence.

Ce travail a été réalisé dans le cadre du projet PEPITES (Processus écologiques et processus $d^{\prime}$ innovation technique et sociale en agriculture de conservation) qui bénéficie du support de I'ANR (Agence nationale de la recherche dans le cadre du programme Systerra.

\section{RÉFÉRENCES BIBLIOGRAPHIQUES}

Ansaloni M., Fouilleux E. (2006). Changement de pratiques agricoles : facteurs et modalités d'hybridation technique des exploitations laitières bretonnes. Économie rurale, $\mathrm{n}^{\circ} 292$, p. 3-17.

Archambeaud M. (2011). Le glyphosate est-il le $4^{\mathrm{e}}$ pilier de l'agriculture de conservation. Techniques culturales simplifiées, $\mathrm{n}^{\circ} 62$, mars-mai, 5 p.

Bardin L. (2007). L'analyse de contenu. Paris, $1^{\text {re }}$ éd. 1977, Presses universitaires de France, 291 p.

Desclaux D., Chiffoleau Y., Nolot J.-M. (2009). Pluralité des agricultures biologiques : enjeux pour la construction des marchés locaux, le choix des variétés et les schémas d'amélioration des plantes. Innovations agronomiques, $\mathrm{n}^{\circ}$ 4, p. 297-306.

FAO (2006). Focus : agriculture de conservation. Département de l'agriculture et de la protection des consommateurs. Organisation des Nations Unies pour l'alimentation et l'agriculture. http://www.fao.org/ag/fr/magazine/0110sp.htm.

FAO (2010). Agriculture de conservation. Département de l'agriculture et de la protection des consommateurs. Organisation des Nations Unies pour l'alimentation et l'agriculture. http://www.fao.org/ag/ca/fr/index.html.
Fleury P. (dir.) (2011). Agriculture biologique et environnement des enjeux convergents. Paris et Dijon, Éducagri Éditions, 272 p.

Goulet F. (2008). Des tensions épistémiques et professionnelles en agriculture. Dynamiques autour des techniques ans labour et leur évaluation environnementale. Revue d'anthropologie des connaissances, $\mathrm{n}^{\circ} 4$, p. 291-310.

Goulet F. (2010). Nature et réenchantement du monde. In «Les mondes agricoles en politique », Presses de la fondation nationale des sciences politiques, p. 51-71.

IFOAM (2004). The Role of Organic Agriculture in Mitigating Climate Change. http:// www.ifoam.org/press/positions/Climate_ study_green_house-gasses.html.

Holland J.-M. (2004). The environmental consequences of adopting conservation tillage in Europe: reviewing the evidence. Agriculture, Ecosystems \& Environment, vol. $103, \mathrm{n}^{\circ} 1$, p. $1-25$.

Kotschi J., Müller-Sämann K. (2004). The Role of Organic Agriculture in Mitigating Climate Change - a Scoping Study. IFOAM Éditions, $11 \mathrm{p}$.

Lamine C. (2011). Anticiper ou temporiser : injonctions environnementales et recompositions des identités professionnelles en céréaliculture. Sociologie du travail, ${ }^{\circ}$ 53, p. 75-92. 
Layton J. (2009). Is conservation agriculture better than organic farming? HowStuffWorks.com. http://science.howstuffworks. com/environmental/green-science/conservation-agriculture.htm.

Leclerc B., Aubert C., Coulombel A., Schreier U. (2007). Chez Friedrich et Manfred Wenz, 25 ans en non-labour! Alter Agri, ${ }^{\circ}$ 82, mars-avril, p. 30-31.

Lémery B. (2003). Les agriculteurs dans la fabrique d'une nouvelle agriculture. Sociologie du travail, vol. 45, $\mathrm{n}^{\circ}$ 1, p. 9-25.

Lémery B., Ingrand S., Dégrange B., Dedieu S. (2005). Agir en situation d'incertitude : le cas des éleveurs de bovins allaitants, Économie rurale, juillet-août, $\mathrm{n}^{\circ} 288$, p. 57-69.

Muller P. (1985). Un schéma d'analyse des politiques sectorielles. Revue française de science politique, vol. 35, n² 2, p. 165-189.

Muller P. (2005). Esquisse d'une théorie du changement dans l'action publique. Structures, acteurs et cadres cognitifs. Revue française de science politique, vol. $55, \mathrm{n}^{\circ} 1$, p. 155-187.

Musselin C. (2005). Sociologie de l'action organisée et analyse des politiques publiques : deux approches pour un même objet? Revue française de science politique, vol. $55, \mathrm{n}^{\circ} 1$, p. 51-71.

Peigné J., Védie H., Demeusy J., Gerber M., Vian J.-F., Gautronneau Y., Cannavaccuiolo M., Aveline A., Giteau L.L., Berry D. (2009). Techniques sans labour en agriculture biologique. Innovations agronomiques, $\mathrm{n}^{\circ}$ 4, p. 23-32.

Peigné J., Ball B., Roger-Estrade J., David C. (2007). Is conservation tillage suitable for organic farming? A review. Soil Use and Management, $\mathrm{n}^{\circ} 23$, juin, p. 129-144.

RMT DevAB (2009). Cultiver sans labour en agriculture biologique. Fiche $\mathrm{n}^{\circ} 3$, agronomie. 4P.

Saikat (2008). The Battle for the Green Label - Organic Farming Versus Conservation Agriculture. AboutMyPlanet.com. http:// www.aboutmy planet.com/green-news/ battle-green-label/
Stassart P.-M., Jamar D. (2009). Agriculture biologique et verrouillage des systèmes de connaissances. Conventionalisation des filières agroalimentaires bio. Innovations agronomiques, $\mathrm{n}^{\circ}$ 4, p. 313-328.

Sylvander B., Bellon S., Benoît M. (2006). Facing the organic reality: the diversity of development models and their consequences on research policies. In Joint Organic Congress Organic Farming and Eur. Rural Development, Odense (DK), 2006/5/30-31.

Thomas F. (2009). AC et AB : des approches complémentaires et convergentes. Techniques culturales simplifiées, $\mathrm{n}^{\circ} 55$, novembre-décembre, p. 3.

Van Dam D., Nizet J., Dejardin M., Streith M. (2009). Les Agriculteurs biologiques : ruptures et innovations. Dijon, Éducagri Éditions, $141 \mathrm{p}$.

Waligora C. (2010). Joseph Pousset - Les Béllières (Orne). Un objectif parmi d'autres : déstocker. Techniques culturales simplifiées, $\mathrm{n}^{\circ} 59$, septembre-octobre, p. 12-13.

Wenger E. (2005). La théorie des communautés de pratique. Apprentissages, sens et identité. Québec, Les Presses de l'Université Laval, 309 p.

\section{Sites internet}

www.agricool.net/

www.agriculture-de-conservation.com/

www.asso-base.fr/

www.institut-agriculture-durable.fr/

www.ecaf.org/

www.fnacs.com/

www.institut-agriculture-durable.fr/

www.agriculture-de-conservation.com/, www.fao.org/ag/ca/fr/index.html www.ifoam.org

www.isara.fr

www.agencebio.org

www.itab.asso.fr

www.fnab.org 